\title{
Mathematical Modeling of the Transport Milk in a Pipeline
}

\section{Yury Tsoy, Vladimir Kirsanov, and Ravza Baisheva}

Federal Scientific Agroengineering Center VIM (FSAC VIM), Federal Budgetary Scientific Institution, Moscow, Russia

\section{Abstract}

The paper presents a mathematical modeling of the process of movement of milk in the pipe in the formation of the slug flow. The estimation of the degree of destabilization of milk fat from hydromechanical factors is presented, which is $6-10 \%$ for the obtained values of the flow rate. Experimental and calculated data show that with the slug flow of transporting milk from the milking unit collector to the upper milk line, the slug speed reaches significant values exceeding $10 \mathrm{~m} / \mathrm{s}$, and the acceleration at the border of sections I and II is $150 \mathrm{~m} / \mathrm{s}^{2}$. All this creates prerequisites for intensive hydromechanical effects on the dispersed composition of fat in milk.

Corresponding Author:

Yury Tsoy

femaks@bk.ru

Received: 25 October 2019

Accepted: 15 November 2019

Published: 25 November 2019

Publishing services provided by

Knowledge E

(c) Yury Tsoy et al. This article is distributed under the terms of the Creative Commons.

Attribution License, which permits unrestricted use and redistribution provided that the original author and source are credited.

Selection and Peer-review under the responsibility of the AgroSMART 2019 Conference Committee.
Keywords: fat globules,slug flow, destabilization, velocity, mass

\section{Introduction}

As it is known, the sections of communications of milk lines, on which the movement of fluid is considered, in most cases can be considered as areas with a constant section along the length (milk hose of the milking machine, technological and transport milk lines, vacuum tubes, etc.) [1--3].

The applied nature of the tasks allows, in relation to the milk lines, considering the movement of a fluid as a one-dimensional motion. By the definition, L.I. Sedov's onedimensional motions are called "motions and processes in which only one geometric coordinate is significant. The word unsteady is added to this name when time $t$ is significant" [4]. The communications of milk lines, with the exception of certain types of technological equipment, as a rule, are cylindrical tubes made of stainless steel, rubber, and polymeric materials. Estimation of the movement mode of liquids shows that practically in all links of the milk lines $\operatorname{Re}>>2320$, which indicates the turbulent character of the flow. The nature of the tasks of engineering calculation and the turbulent motion of a fluid provide a basis for analyzing the flow characteristics averaged over the cross section.

\section{G OPEN ACCESS}




\section{Methods and Equipment}

Slug flow is quite typical for milk hoses when milking in stalls into the milk line and for the milk line washing mode, where the presence of air and water plugs is one of the main factors determining the washing efficiency $[5,6]$. For the analysis, we will accept the following assumptions: "slug" is a continuous body; liquid is incompressible; the mass of the tube is constant. The adoption of a "continuous model" in the study of the cork mode of movement of gas-liquid mixtures is, according to V.A. Mamaev et al., the most common approach [7]. The mass of the milk slug when moving in reality does not remain constant. Thus, the movement of the milk slug along the milk hose into the milk line is usually accompanied by some leakage of fluid back down. However, given that the main goal is to assess the effect of flow velocity parameters on the dispersed fat composition, then at the first stage such an assumption seems to be justified.

Taking into account the accepted assumptions, we write the equation of motion of the center of mass of the milk slug relative to the longitudinal axis of the pipe in the following form

$$
\alpha_{m} \frac{d V}{d t}=P_{1}-P_{2}-c_{T} \sin \beta-\tau_{s h r} \chi \ell
$$

where $m$-- weight slug;

$P_{1}=p_{1} \omega$ and $P_{2}=p_{2} \omega$-- the total pressure on the tube from the bottom and top;

$V$-- the average velocity slug of section;

$C_{T}=m g$-- heft slug;

$\omega$ and $\ell$-- section and length of slug;

$X$-- wetted perimeter;

$\mathrm{T}_{\text {shs }}{ }^{--}$shear stress;

$\beta$-- pipe angle;

$\alpha$-- amount of movement, similar in meaning to the Coriolis coefficient;

$P_{1}$ and $P_{2}$-- top and bottom pressure of slug.

In equation (1)we replace $\sin \beta, \mathrm{T}_{s h r}, \mathrm{X}$, taking into account the following values

$$
\sin \beta=\frac{z_{2}-z_{1}}{\ell} ; \tau_{s h r}=\lambda_{s h r} \rho \frac{V^{2}}{8} ; \frac{\omega}{\chi}=R_{h y d}=\frac{d}{4},
$$

where $z_{1}$ and $z_{2}$-- geometric heights.

Next, (2) substituting in (1) and dividing the resulting expression we will have $\omega \gamma$

$$
\frac{P_{1}-P_{2}}{\gamma}-z_{2}+z_{1}=\lambda \frac{\ell V^{2}}{d 2 g}+\alpha \frac{\ell}{g} \frac{d V}{d t} .
$$


Equation (3) can be represented as follows:

$$
C_{1} V^{2}+C_{2} \frac{d V}{d t}=C(t)-z_{2}+z_{1}
$$

where $c_{1}, c_{2}$ and $c(t)$ have the same meaning as in the previously considered equation. Really $c(t)=\frac{P_{1}-P_{2}}{\gamma}$-- there is a pressure differential providing the movement of the slug in general case changing in time; $c_{1}=\lambda_{C T} \frac{\ell}{d}$-- drag coefficient; $c_{2} \frac{d V}{d t}$-- is none other than the inertial pressure; $c_{2}=\frac{\alpha \ell}{g}$.

In general, the pressure drop varies with time. When $c(t)$ is constant, the integration of equation (4) gives the expressions for the velocity and acceleration of a moving plug that are similar to the previous case by the form of the expression

$$
\begin{aligned}
& V_{n}=N\left[\frac{e^{t / M}-1}{\ell^{t / M}+1}\right] ; \\
& j_{n}=\frac{2 N}{M} \cdot \frac{\ell^{t / M}}{\left(e^{t / M}+1\right)^{2}},
\end{aligned}
$$

where $N=\sqrt{\left(\frac{\Delta P-\ell \sin \beta}{\ell}\right) \frac{2 g d}{\lambda}} ; M=\frac{\alpha_{0} \ell d}{\sqrt{2 \ell \lambda_{C T} d g(\Delta P-\ell \sin \beta)}} ; \ell \cdot \sin \beta=z_{2}--z_{1}$.

The experiments have shown that under the slug flow movement of milk in the milk claw, the pressure drop is as follows: in Figure 1. As it can be seen on the graph of changes in pressure drop, we can distinguish three characteristic sections $t_{1}, t_{2}, t_{3}$. In sections $t_{1}$ and $t_{3}$, it is conditionally possible to assume a linear dependence of the pressure drop as a function of time, and in section $t_{2}$, the pressure drop can be considered constant. Such an idealized scheme for changing the pressure drop is used in the analysis of the operation of milking machines [8]. On the basis of the idealized scheme in the segment $t_{1}$, can take $c(t)=k_{1} t_{1}$; on the segment $t_{2} c(t)=$ const $=c_{\text {max }}$; on the segment $t_{3} c(t)=c_{o}-k_{2} t_{3} \cdot k_{1}$ and $k_{2}$-- here the slopes of the linear dependencies

When $c(t)=k_{1} t$ equation (4) will take the following form

$$
c_{1} V^{2}+c_{2} \frac{d V}{d t}=k_{1} t-\ell_{n} \sin \beta
$$

or by typing the notation

$$
\begin{gathered}
a_{1}=\frac{c_{1}}{c_{2}}=\lambda \frac{\ell}{d} \cdot \frac{g}{\alpha \cdot \ell}=\frac{\lambda g}{d \alpha} ; a_{2}=\frac{k_{1}}{c_{2}}=\frac{k_{1} g}{\alpha \cdot \ell_{n}} ; \\
c_{0}=\frac{\ell_{n} \sin \beta \cdot g}{\alpha \cdot \ell_{n}}=\frac{g \sin \beta}{\alpha} .
\end{gathered}
$$

The resulting equation can be rewritten in the following form

$$
\frac{d V}{d t}=a_{2} t-a_{1} V^{2}-c_{0} .
$$


The solution of the equation will be sought in the form of a power series

$$
V(t)=V(0)+\frac{V^{\prime}(0)}{1 !} t+\frac{V^{\prime \prime}(0)}{2 !} t^{2}+\frac{V^{\prime \prime \prime}(0)}{3 !} t^{3} \ldots
$$

Under the following initial conditions $t=0 ; V=0 ; V^{\prime}=--c_{0}$.

Here $V^{\prime}=-c_{0}$ means that at the initial moment the acceleration of gravity acts on the tube. From (8) we find that $V^{\prime \prime}(0)=a_{2}$. Then as a first approximation can write

$$
V(t)=-c_{0} t+\frac{a_{2}}{2} t^{2} .
$$

When analyzing the movement of the milk slug in the second section, it must be borne in mind that when $t_{I I}=0, v_{I I}(0)=V_{I k}$. Therefore, solutions of equation (5) with $c(t)=$ const will have the following form:

$$
\begin{gathered}
V=N\left[\frac{e^{\frac{t_{I I+t_{f}}}{m}}-1}{e^{\frac{t_{I I+t_{f}}}{m}}+1}\right] ; \\
S=2 M N \ln \left(\frac{1+e^{\frac{t_{I I+t_{f}}}{m}}}{2}\right)-2 M N\left(t_{I I}+t_{f}\right)-S_{f} .
\end{gathered}
$$

where $t_{f}$ is the time during milk slug, starting with $t=0, V_{I I}=0$ will pick up speed, numerically equal to $V_{I I}=V_{I k}$, i.e. speed at the border of the first section. $S_{f}$-- the path that will pass the milk slug during $t_{f}$ at zero initial speed. The value of $t_{f}$ we find from the next equation:

$$
t_{f}=M \ln \left[\frac{N+V_{I k}}{N-V_{I k}}\right]
$$

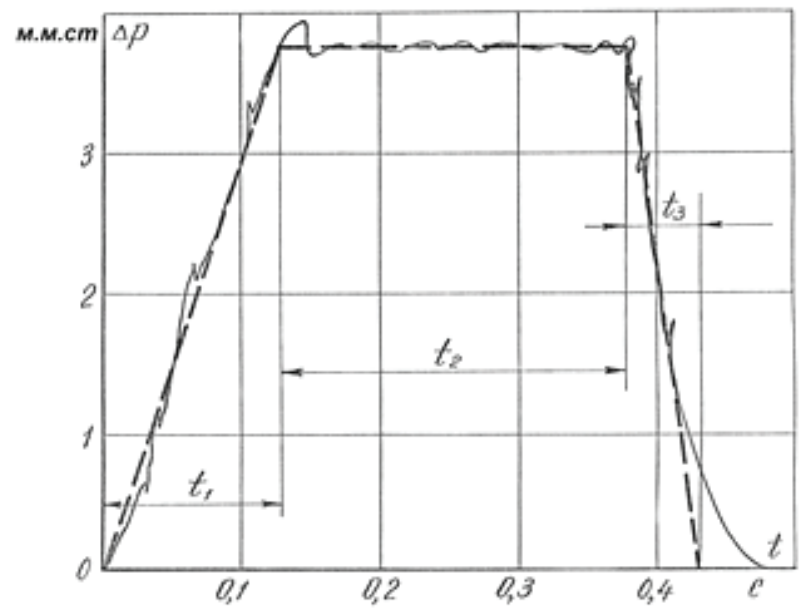

Figure 1: Changing the pressure drop of slug flow.

The initial conditions of the third section of the movement of the milk slug we write in the following form

$$
t_{I I I}=0 ; V_{I I I}(0)=V_{I I k} ; V_{I I I}^{\prime}(0)=0 ;
$$


where $V_{I I k}$-- velocity milk slug in the II section end.

Milk slug of movement equation:

$$
\frac{d V}{d t}=P_{0}-a_{2} t-a_{1} V^{2}-c_{0} .
$$

where $P_{0}=\frac{\Delta H}{c_{2}}$.

From (14) will find V"(0); V"(0) = -- $\mathrm{a}_{2}--2 \mathrm{aVV} \mathrm{V}^{\prime}=--\mathrm{a}_{2}$

Then taking, as a power series solution (9), taking into account the initial conditions (13)

$$
V_{I I}(t)=V_{I I k}-\frac{a_{2}}{2} t^{2} ; S_{I I I}(t)=V_{I I k} t-\frac{a_{2}}{6} t^{3} .
$$

When analyzing the slug flow of milk movement, an assumption was made about the constancy of the mass of the cork. In fact, as already indicated, the movement of the milk slug, especially on inclined and vertical sections, is accompanied by some leakage of fluid along the wall in the direction opposite to the motion of the slug. With relatively large tube lengths, the change in mass of the slug may be significant. To describe the movement of the milk slug with "mass loss", we use the Meshchersky equation [9].

$$
M \frac{d V}{d t}=R+\left(u_{1}-V\right) \frac{d M}{d t},
$$

where $\mathrm{M}$-- weight slug;

$\mathrm{V}$-- velocity slug;

$\mathrm{R}$-- resultant of all forces;

$\mathrm{u}_{1}$-- absolute velocity of the reflected particles.

In accordance with (1) the resultant of forces applied to the slug as it moves up, it will be equal to

$$
R=\left(P_{1}-P_{2}\right) \omega-M g \sin \beta-M \frac{\lambda}{d} \cdot \frac{V^{2}}{2} .
$$

Then, taking into account the obtained $\mathrm{R}$ value, the Meshchersky equation, with reference to the milk slug, will take the following form

$$
M \alpha \frac{d V}{d t}=\left(P_{1}-P_{2}\right) \omega-M g \sin \beta-M \frac{\lambda}{d} \cdot \frac{V^{2}}{2}+\left(u_{1}-V\right) \frac{d M}{d t} .
$$

The general solution of such equations for the case of inverse problems when $V(t)$ is given, A. Kosmodemyansky results in the following form [10]:

$$
M=e^{\frac{-1}{V} \int\left(g+\frac{d V}{d t}\right)}\left[c-\frac{k}{V_{1}} \int V^{2} e^{\int \frac{1}{V_{1}}\left(g+\frac{d V}{d t}\right) d t} d t\right],
$$

Where $c$-- integrating constant. 
With the known law of body motion, relation (19) allows determining the law of change in $M(t)$. To solve the direct problem, it is necessary to do the opposite, specifying $M(t)$ to determine $V(t)$. The loss of mass of the slug depends on the slick thickness remaining on the inner wall of the communications. Therefore, knowing the slick thickness or the law of change, in principle, it is possible to determine the magnitude of the loss of the mass of the slug. In [11], the problem of a liquid slick formed on the surface of a body extracted from a liquid was considered. It is established that in the general case the slick thickness is proportional $\sqrt{\frac{M V}{\rho g}}$, where $V$ is the speed of the slug. In this case, the formula for determining $\Delta \mathrm{M}$ takes on a very complex form.

$$
\Delta M=\pi d h(V) S=\pi d h(V) \int V(t) d t,
$$

where $h(V)$-- slick thickness dependence on the speed;

$S=\int V(t) d t$-- the length traveled by the slug, which in itself, depends on the desired function $V(t)$.

Therefore, it is advisable here to use one of the approximate numerical methods, for example, the piecewise approximation method, with assumptions made at the sites that the loss of mass is proportional only to the distance traveled. Note, as it will be shown below, that such an assumption turned out to be quite convenient even in practical use for determining the mass loss of a slug of washing liquid during washing. Knowing the length of the milk pipeline and the specific "loss" of mass per unit length, it is easy to calculate the original length $[12,13]$. The thickness of the slick at the beginning and end of the process can be calculated from the data of the work.

To test equation (7) describing the slug flow, it was necessary to use another method -- high-speed filming, since the method used gives large errors due to the relatively small volumes of the liquid slug itself and the presence of the opposite milk flow along the wall $[14,15]$. The scheme of the experimental setup for the study of the slug flow is shown in Figure 2. At the lower end of the dairy communication, an adjustable air intake throttle 2 is installed. The opening of the throttle is a valve with an electromagnetic drive.

The change in the intensity of milk intake is carried out by the crane 1. The area of the dairy line was imitated by a transparent glass tube $\mathrm{d} \approx 15 \mathrm{~mm}$, at the ends of which a driving differential was created due to vacuum. The movement of the milk slug inside the glass tube was fixed on the film by a high-speed camera. The frame rate is 2500 fps.

In parallel, the pressure in the initial and final points of the milk line was recorded using a strain gauge 6 , an amplifier and a recorder 10 . According to the obtained film material, 
the dependence $S=f(t)$ was determined, and further, by graphical differentiation, the dependences $V=f(t)$ and $j=f(t)$.

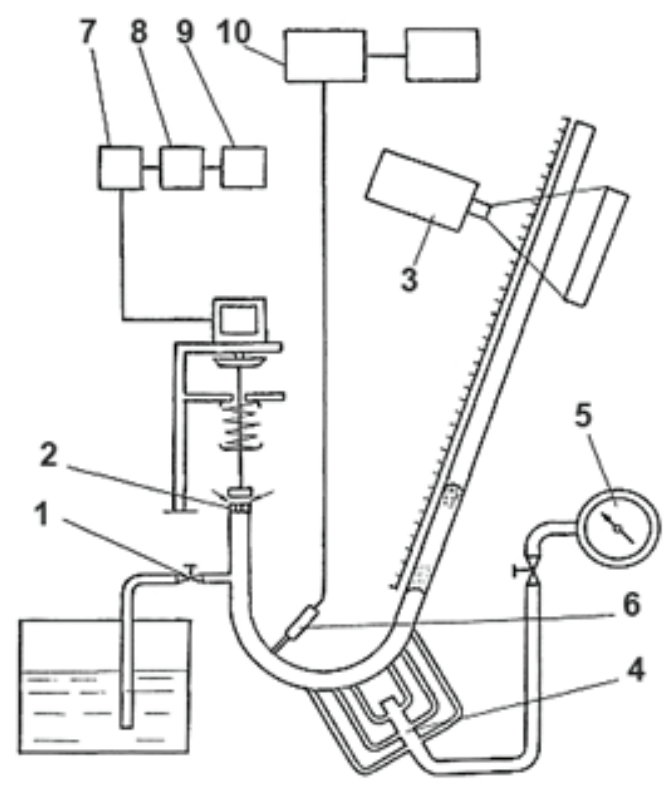

Figure 2: Scheme of the experimental setup for the study of milk slug flow: 1 -- crane; 2 -- adjustable air intake throttle; 3 -- high-speed video camera; 4 -- air distributor with needles; 5 -- gas meter; 6 -- strain gauge; 7, 8, 9 -- respectively the breaker, electric stopwatch and pulse counter; 10 -- tensoamplifier.

\section{Results}

Figure 3 presents the experimental and calculated dependences $V=f(t)$ and $S=f(t)$ for the slug flow with a variable pressure drop characteristic of a number of nodes of milk lines. The change in pressure drop wore a trapezoidal character with three sections of duration of $0.12 \mathrm{~s}, 0.08 \mathrm{~s}$, and $0.12 \mathrm{~s}$. The ratio between the plots was changed by adjustable chokes and the magnitude of the vacuum. The length of the milk tube was changed by changing the intensity of milk intake. In Figure $3, \ell=0.25 \mathrm{~m}, \Delta \mathrm{P}=3.8 \mathrm{~m}$, $\sin \beta=0.72, d=1.4 \cdot 10^{-2} \mathrm{~m}, \mathrm{k}=31.5, \mathrm{a}_{2}=1.24 \cdot 10^{-3}, \alpha_{0}=1$ are shown. It can be seen from the graphs that the theoretical dependences in general have good agreement with the experimental ones and correctly reflect the process of moving the milk slug.

The experimental and calculated data show that with the slug flow of transporting milk from the milking unit collector to the upper milk line, the "slug" speed reaches significant values exceeding $10 \mathrm{~m} / \mathrm{s}$, and the acceleration at the border of sections I and II is $150 \mathrm{~m} / \mathrm{s}^{2}$. All this creates prerequisites for intensive hydromechanical effects on the dispersed composition of fat in milk. 


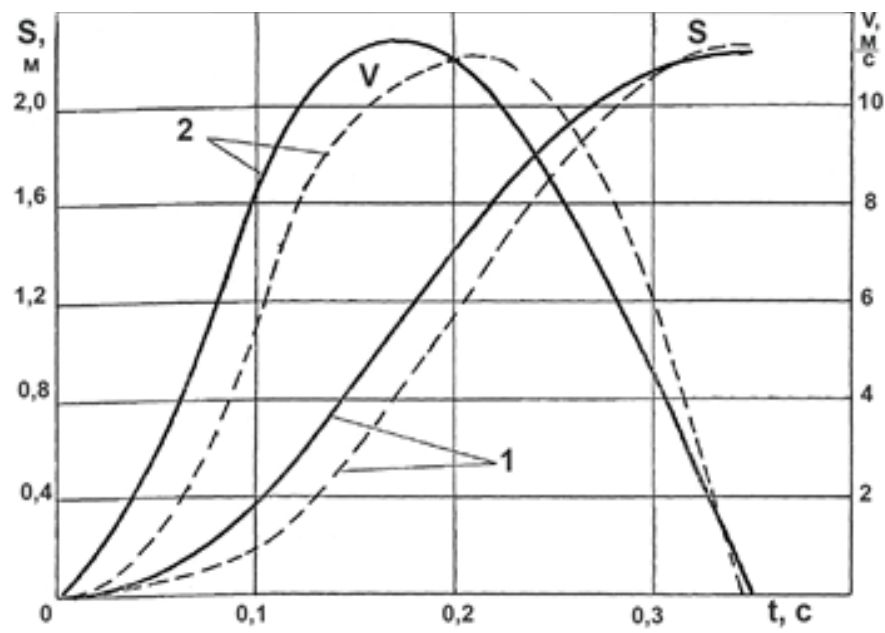

Figure 3: Dependencies $S(t)$ and $V(t)$ in the slug flow of milk pipeline: dashed line --theoretical;solid line --experimental.

a)

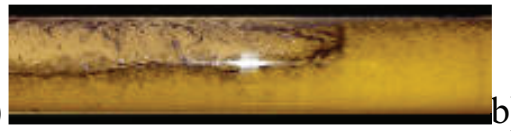

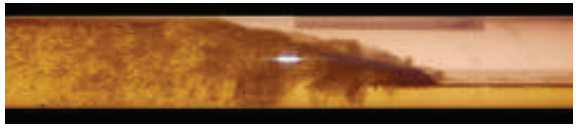

Figure 4: Liquid slug movement: a-after part; b-front part.

\section{Discussion}

The calculated values of $S=f(t)$ differ from the experimental data by an average of $15 \%$, which, if we take the errors arising from the processing of film materials, can be considered quite acceptable. These discrepancies, of course, were influenced by such facts as the intensive saturation of the stern and fore part of the slug with air, which is clearly seen in the photograph (Fig. 4) and the decrease in the mass of the slug during movement due to the reverse flow of fluid near the pipe wall due to border effects. In addition it is necessary to take into account that the relatively large error, which gives the method of graphical differentiation, is used to build experimental dependencies $V$ $=f(t)$ and $j=f(t)$.

\section{Conclusion}

The evaluation of the destabilization of milk fat only from hydromechanical vortex factors (without taking into account the effects of air) showed that, with the obtained values of the flow velocity, it is $6-10 \%$. In addition, large values of velocity gradients create conditions for intensification under the action of inertial forces of mutual displacements and collisions of fat particles and, as a consequence, the formation of oil conglomerates. 


\section{Funding}

This work was supported by State program of the Ministry of Science and Higher Education of the Russian Federation of Federal Budgetary Scientific InstitutionFederal Scientific Agroengineering Center VIM (FSAC VIM) under State Program No. [0581-20190009 of 06/14/2018].

\section{Acknowledgement}

The authors would like to thank their colleague for their contribution and support to the research. They are also thankful to all the reviewers who gave their valuable inputs to the manuscript and helped in completing the paper.

\section{Conflict of Interest}

The authors have no conflict of interest to declare.

\section{References}

[1] Allcock, J., Postma, E. (2013). Understandingdynamicmilkingmachinetests. JournalLivestock, vol. 18, iss. 2, pp. 24--29. DOI: org/10.12968/live.2013.18.2.24

[2] Wagner, A.M., Ruegg, P.L. (2002). TheEffectofManualForestrippingonMilkingPerformanceofHolsteinDairyCows. JournalofDairyScience, vol. 85, no. 3, pp. 1--6.

[3] Upton, J., Reinemann, D.J., Penry, J.F., Thompson, P.D. (2016). A quarter milking analysis device e Development and demonstration. JournalBiosystemsengineering, pp. 259--264.

[4] Sedov, L.I. (1973). Continuum mechanics, vol. I. Moscow: Science, 384 p.

[5] Gonzalez-Mejia, A., Styles, D., Wilson, P., Gibbons, J. (2018). Metricsandmethodsforcharacterizingdairyfarmintensificationusingfarmsurveydata. PLOS ONE, no. 13(5), e0195286. Retrievedfrom: https://doi.org/10.1371/journal.pone.0195286

[6] Matthews, C.A., Shaw, J.M., Earl, W. (2017). Theeconomyandefficiencyof a milkingmachine. IOWA StateUniversity, vol. 21, no. 248, pp. 1--24.

[7] Mamaev, V.A., Odisharia, G., Klapchuk, O.V. et al. (1978). The movement of gas-liquid mixtures in pipes. Moscow, Nedra, 270 p.

[8] Melnikov, S.V. (1985). Technological equipment of livestock farms and complexes. Leningrad:Agropromizdat, $640 \mathrm{p}$. 
[9] Meschersky, I.V. (1952). Works on the mechanics of bodies of variable mass. Moscow: Gostekhizdat.

[10] Kosmodemyansky, A.A. (1966). The course of theoretical mechanics, part II. Moscow, pp. 70--72.

[11] Levich, V.G. (1952). Physico-chemical hydrodynamics. Moscow, p. 498--524.

[12] Sanchis, A., Johnson, G.W., Jensen, A. (2011) The Formation of Hydrodynamic Slugs by The Interaction of Waves In Gas-Liquid Two-Phase Pipe Flow, Int. Journal Multiphase Flow, vol. 37, no. 4, pp. 358--368.

[13] Fonseca, Jr.R., Barras, Jr.J.M., Azevedo, L.F.A. (2009). Liquid Velocity Field and Bubble Shape Measurements in Two-Phase, Horizontal Slug Flow. Proceedings of COBEM 2009. Gramado, RS Brazil, COB09-245.

[14] Carneiro, J.N.E., Fonseca Jr. R., Ortega, A.J., Chucuya, R.C., Nieckele, A.O., Azevedo, L.F.A. (2011). Statistical characterization of two-phase slug flow in a horizontal pipe. Journal of the Brazilian Society of Mechanical Sciences and Engineering, no. 33(spe1), pp. 251--258. Retrieved from: https://dx.doi.org/10.1590/ S1678-58782011000500007

[15] Want, X., Guo, L., Zhang, X., (2007). An Experimental Study of the Statistical Parameters of Gas-Liquid Two-Phase Slug Flow in Horizontal Pipeline. Int. Journal Heat Mass Transfer, vol. 50, pp. 2439--2443. 